\title{
Challenges in Continuum Modelling of Intergranular Fracture
}

\author{
V. R. Coffman*, J. P. Sethna ${ }^{\dagger}$, A. R. Ingraffea ${ }^{\ddagger}$, J. E. Bozek ${ }^{\ddagger}$, N. P. Bailey ${ }^{\S}$ and \\ E. I. Barker ${ }^{\text {It }}$ \\ *Information Technology Laboratory, National Institute of Standards and Technology, 100 Bureau Drive, Mail Stop 89I0, Gaithersburg, \\ MD 20899, USA \\ †Laboratory of Atomic and Solid State Physics (LASSP), Clark Hall, Cornell University, Ithaca, NY I4853-250I, USA \\ ${ }^{\ddagger}$ Cornell Fracture Group, Rhodes Hall, Cornell University, Ithaca, NY I4853-250 I, USA \\ §Department of Mathematics and Physics (IMFUFA), DNRF Center 'Glass and Time', Roskilde University, PO Box 260, DK-4000 \\ Roskilde, Denmark \\ ILLos Alamos National Laboratory, Los Alamos, NM 87545, USA
}

\begin{abstract}
Intergranular fracture in polycrystals is often simulated by finite elements coupled to a cohesive zone model for the interfaces, requiring cohesive laws for grain boundaries as a function of their geometry. We discuss three challenges in understanding intergranular fracture in polycrystals. First, 3D grain boundary geometries comprise a five-dimensional space. Second, the energy and peak stress of grain boundaries have singularities for all commensurate grain boundaries, especially those with short repeat distances. Thirdly, fracture nucleation and growth depend not only upon the properties of grain boundaries, but also in crucial ways on edges, corners and triple junctions of even greater geometrical complexity. To address the first two challenges, we explore the physical underpinnings for creating functional forms to capture the hierarchical commensurability structure in the grain boundary properties. To address the last challenge, we demonstrate a method for atomistically extracting the fracture properties of geometrically complex local regions on the fly from within a finite element simulation.
\end{abstract}

KEY WORDS: finite element analysis, fracture, molecular dynamics, multiscale modelling, polycrystals

\section{Introduction}

The nucleation and propagation of cracks in practical engineering materials depends strongly on the mesoscopic structure; grain boundaries, polyphase inclusions, dislocations and other defects determine the toughness. Can continuum computational modelling be used to quantitatively study such complex failure modes?

Consider brittle intergranular fracture-rupture at the boundary between two crystallites. Ignore for the moment problems like embrittlement caused by impurity segregation to grain boundaries, and assume a clean, single-phase, equilibrium grain boundary. Direct atomistic simulations are infeasible for anything larger than nanocrystals; even simulations that focus on the boundaries [1] will be overwhelmed by the number of relevant atoms for systems larger than microns in scale. Hence, let us imagine a finite element simulation of the polycrystal coupled to, say, cohesive zone models (CZM) for each interface [2-5]. (A cohesive law gives the crack opening as a function of the traction across the interface; it is often parameterised by a peak stress and a total energy associated with cleaving [6].) Can one use atomistic models to measure the fracture properties of the individual boundaries, and then use these properties in a realistic continuum fracture simulation?

Previous CZM simulations of polycrystals have used cohesive laws that are guessed, chosen for numerical convergence, and do not take into account the effect of varying grain boundary geometries within the material. The same cohesive law is used throughout the material despite the fact that in a real material, the geometries of the grain boundaries/ phase interfaces must vary [7-9]. Yamakov et al. [10, 11] describe a method for calculating cohesive laws atomistically by calculating the stresses and displacements in $3 \mathrm{D}$ cracks propagating in ductile $\mathrm{Al}$ for one grain boundary geometry and incorporate this method into multiscale simulations of intergranular 
fracture. Zhou et al. [12] use molecular dynamics (MD) to derive cohesive laws for the fracture between two brittle materials under mixed-mode loading conditions.

We outline here some serious challenges involved in continuum modelling of intergranular fracture: the geometrical complexity of three-dimensional grain boundaries, singularities at all commensurate grain boundaries and the important role of features such as triple junctions of grains that are not described by planar cohesive laws [6,13-15]. We then suggest a new approach to overcome these challenges: using direct atomistic simulations of local regions of interest and describe a corresponding open source software tool.

\section{Geometrical Complexity}

The first challenge is that of geometrical complexity. The cohesive law will depend on the structure of the grain boundary. The macroscopic geometry of a 3D grain boundary depends on five parameters that describe the relative orientations of the two grains. (The fracture dynamics may in principle depend on properties of the crack that are not treated explicitly by the CZM, such as the orientation of the crack front within the grain boundary or the three separate stress intensity factors.) The atomistic structure also depends on how the two crystal lattices are translated with respect to one another along the three directions, which can greatly affect the pattern of atoms along the boundary and hence the peak stress and energy [13]. One particular shift will constitute a global energy minimum corresponding to the most natural configuration [13].

In a polycrystal, one grain will have to find an energy-minimising configuration with several other, neighbouring grains. For a particular grain boundary in a polycrystal, where each grain has been pinned by other neighbouring grains, there will be a competition between elastic straining and plastic deforming. For thick grains, in equilibrium, and away from intersections, one can show that it is advantageous for the crystallites to strain slightly to allow the boundary to find the global energy minimum.

To compute the cohesive properties of grain boundaries efficiently, it is useful to use periodic boundary conditions in directions perpendicular to the grain boundary, which demands that the two crystals have finite repeat distances along the interface and that the repeat distances be commensurate with one another. We have found a systematic method of finding commensurate grain boundaries [13] and have also generalised it to allow for slight

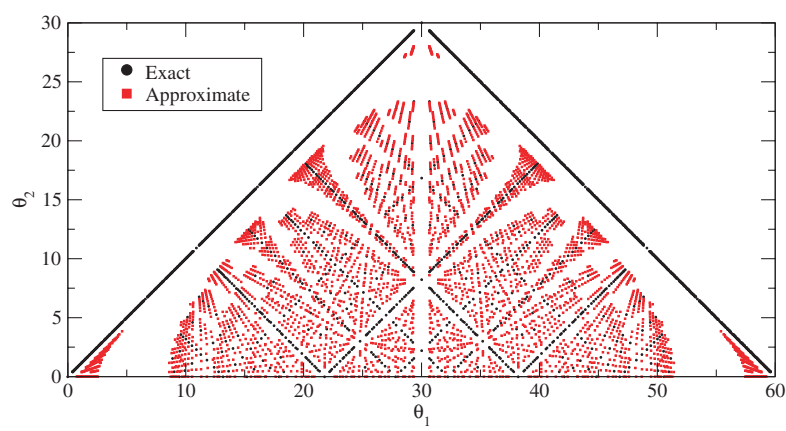

Figure I: Commensurate 2D Grain Boundaries. The set of points above represent all (105) 2D grain boundary geometries that can be simulated in a periodic box of 70 lattice constants or less, with a strain of $0.05 \%$ or less. $\theta_{1}$ and $\theta_{2}$ are the tilt angles that define the grain boundary geometry. The black dots represent geometries that are exactly rational while the gray dots represent geometries that approximate commensurate grain boundaries. There are gaps near perfect crystals, symmetric grain boundaries, and high symmetry grain boundaries because creating a new, nearby geometry requires adding flaws (extra dislocations) at large separations [13]

elastic strains to mesh the two crystal boundaries together. We can approximate commensurate grain boundaries by allowing small strains in either direction. Figure 1 describes the commensurate grain boundaries for 2D hexagonal crystals, Figure 2 shows a cross section of the five-dimensional space of commensurate and near commensurate grain boundaries for three-dimensional face centered cubic crystals.

Not only are these commensurability questions of practical importance in efficiently computing the properties of grain boundaries, commensurate grain boundaries (especially those with short repeat distances) also have especially low energies and high

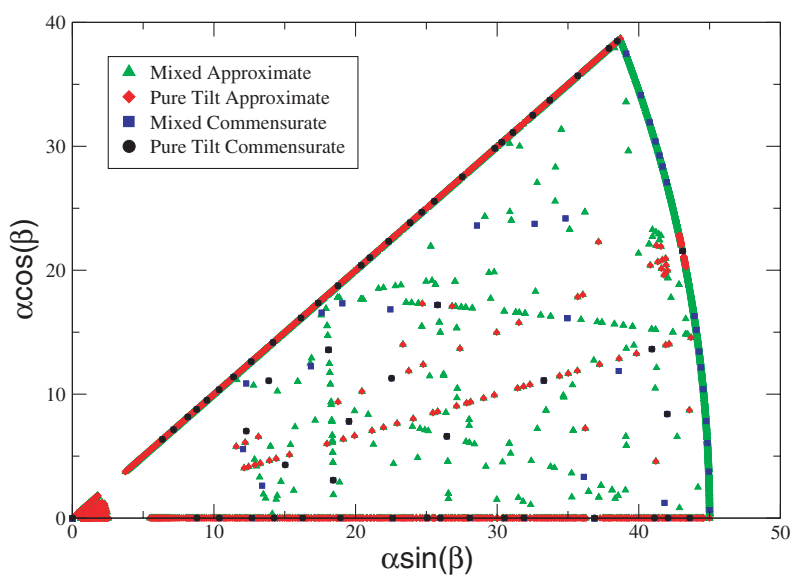

Figure 2: Commensurate 3D Grain Boundaries. The set of points above represent a stereographic projection [defined for normal vector $\vec{n}$ by $\vec{n}=(i, j, k) / \sqrt{i^{2}+j^{2}+k^{2}}=(\sin \alpha \cos \beta, \sin$ $\alpha \sin \beta, \cos \alpha)$ ] of all face centered cubic surface orientations that are commensurate with the (100) surface within an area $<100$ square lattice constants with a strain of $0.1 \%$ or less. This constitutes a three dimensional cross section of the five dimensional space of grain boundary geometries, where the twist angle parameter has been collapsed onto a 2D plot 
peak stresses $[13,16]$. Modelling the geometry dependence of the peak stress and energy could be relatively straightforward if they depended in comprehensible ways on the five geometrical parameters.

\section{Singularities at Commensurate Grain Boundaries}

The second challenge in continuum models of fracture is to incorporate the singularities associated with commensurate geometries into appropriate functional forms. It is well established that cusp singularities in the energy occur at special high-symmetry grain boundaries with low repeat distances [13, 1619]. The cusps in energy can be understood by thinking of a high-symmetry boundary as an undeformed reference crystal [13]. Nearby grain boundaries (whose crystallites are rotated by a small angle $\theta$ from the high-symmetry boundary) are thus described by decorating the high-symmetry boundary with a few extra dislocations, just as a low-angle grain boundary in a crystal can be described as an array of well-separated dislocations. This analogy leads to a functional form for grain boundary energy as a function of tilt angle in which the cusps around the special high-symmetry grain boundaries have the same $\theta \log \theta$ form as low-angle grain boundaries [13]. Figure 3A shows the results for a systematic study of symmetrical 2D grain boundaries and the resulting fitting function [13].

For the peak stress, it is known that there are jumps at the same special grain boundaries $[13,16,18]$. We can also understand these jumps by using the dislocation picture described above [13]. As we add a dislocation to the high-symmetry grain boundary, we add a nucleation point for fracture, causing a discontinuity in the peak stress. As a result, the plot of peak stress versus tilt angles is discontinuous at every commensurate geometry (Figure 3B). By considering the elastic interaction between the extra dislocations, we have been able to understand also the dependence of the peak stress in the vicinity of the highsymmetry boundaries (see Figure 3B and [13]).

\section{Importance of Local Geometries}

Are cohesive laws enough? We have studied this question computationally [6] by comparing a direct atomistic simulation of polycrystalline fracture with a finite element simulation of the same geometry using cohesive law parameters derived from the same interatomic potential. Figure 4 shows a snapshot of the two simulations of polycrystalline fracture in
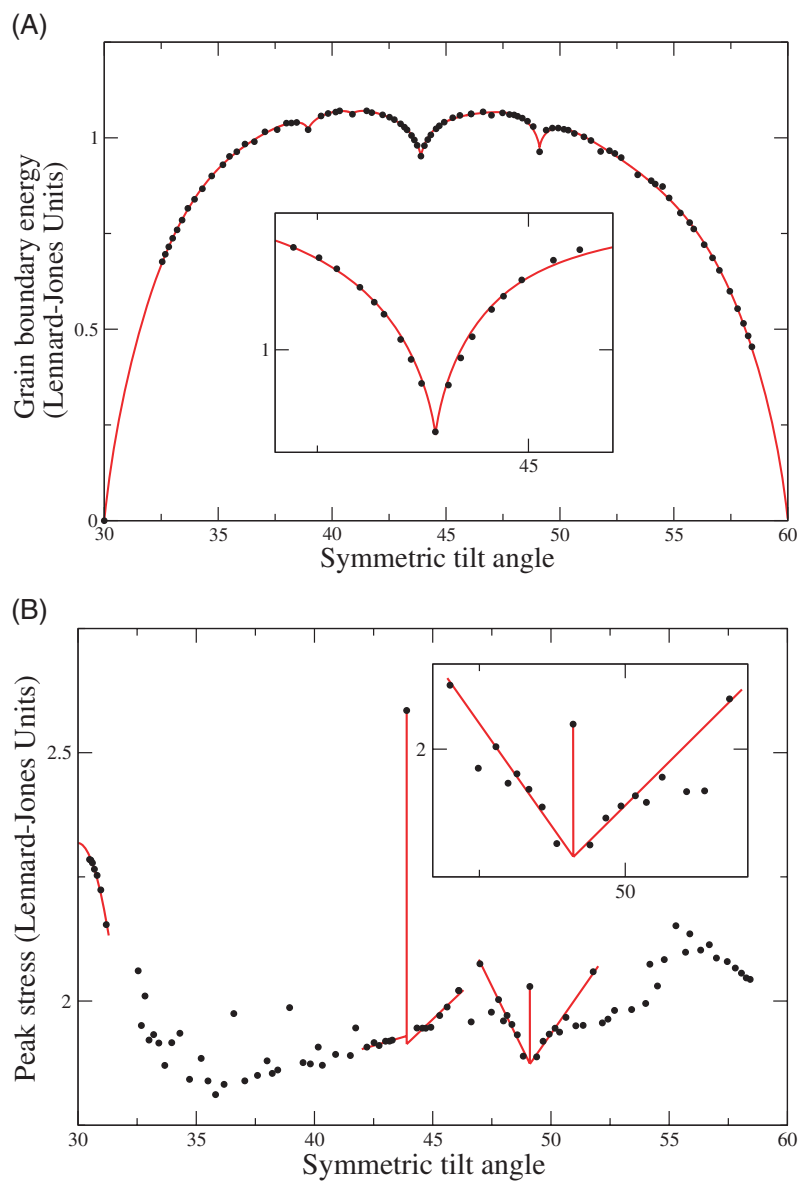

Figure 3: Singularities at Special Grain Boundaries. Cusps in energy appear at high symmetry grain boundaries (A) and have the same $\theta \log \theta$ shape as the energy of low angle grain boundaries. The red line is the functional form described in [13]. The peak stress as a function of tilt angle (B) is discontinuous everywhere, with higher values at special tilt angles corresponding to high symmetry grain boundary geometries. The dependence of peak stress on angle near the high symmetry grain boundaries (lines and parabolas shown) can be explained using the interactions of the extra dislocations added [13]. The inset shows the peak stress for the grain boundary with tilt angle 49.1 and the nearby geometries

Stillinger-Weber silicon. Both in this case and for other simulations, the atomistic simulations fail at significantly lower stresses than the continuum simulations. Crack nucleation in both atomistic and continuum simulations happens not only in the middle of grain boundaries, but also at the triple junction lines, edges and corners which are not quantitatively described by the cohesive laws for the grain boundary interfaces. Similarly, quantitative understanding of how the crack turns, branches or goes intragranular (Figure 4) at triple junctions demands that we understand the effects of the irregular atomistic configurations at these junctions.

The third challenge is thus to develop an effective computational method for modelling more complex local geometries. One in principle could incorporate an analytical understanding of these local geometries 
(A)

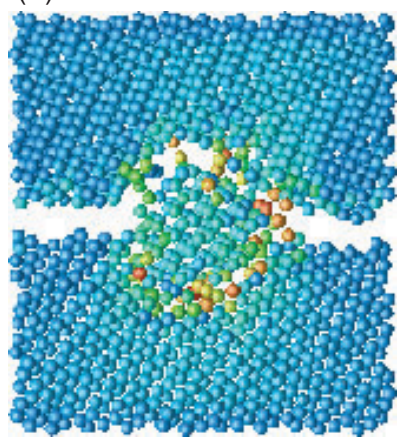

(B)

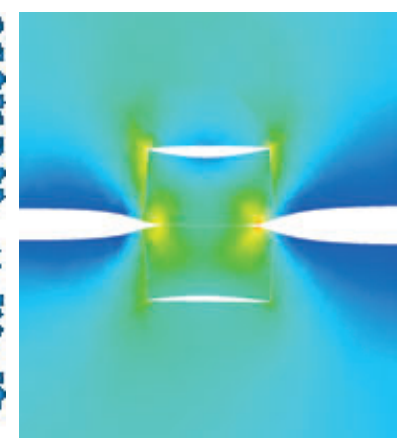

(C)

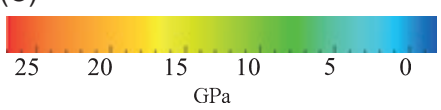

Figure 4: Comparison of Atomistic and Continuum Fracture Simulations. The figures above show a center cross-section of the atomistic (A) and continuum (B) polycrystal simulations. The geometry is that of a cube in cube with three crystal orientations for the upper half of the outer cube, lower half of the outer cube, and the inner cube. An upwards displacement is imposed on the top face. The color scale indicates the vertical component of stress. In the FEM simulation (B), we include an interface through the center of the inner cube to allow for intragranular fracture. The atomistic simulation fails at a strain 0.02 smaller than the FEM simulation (a percentage error of $20 \%$ ), with the crack propagating partially intragranularly through the inner cube and partially through the interface at the top of the inner cube. The continuum simulation cracks straight through the center plane of the inner cube [6]

into, for example, cohesive laws for triple junctions, but the geometrical complexity would seem daunting. Can one rely here on direct atomistic simulations? Brittle crack nucleation is a local phenomenon, and the intersection of a growing crack with a triple junction edge again will happen at a point. A feasible atomistic simulation of the local region of interest could be launched whenever the continuum simulation reached a stress state where its cohesive laws become unreliable. The information about the local geometry (elastic strains, grain orientations and impinging crack surfaces) would be transferred from the continuum simulation to generate the atomistic configuration, and the results of the atomistic simulation (nucleation thresholds, crack branching and turning events) passed back to the finite element simulation.

\section{Overlapping Finite Elements and Molecular Dynamics}

To address this third challenge, we have developed a tool called Overlapping Finite Elements and Molecular Dynamics (OFEMD), which uses the DigitalMaterial [20] atomistic simulation environment to perform MD simulations within a finite element mesh. An example of a simulation of fracture at a triple junction of grains generated automatically by OFEMD is shown in Figure 5. In this example, the finite element model (FEM) is that of a polycrystal generated with a Voronoi tessellation, and we have used the MEAM potential [21, 22] to model Si.

Overlapping Finite Elements and Molecular Dynamics retrieves information about the geometry, orientation and boundary strains from a finite element simulation by communicating with a remote XML repository or database via WebServices or SQL $[23,24]$. The user has the option to decorate a local region of interest centered on an arbitrary point, mesh feature (vertex, edge or face) or the entire mesh to perform an overall MD to FEM comparison as in [6]. For decorating local regions of interest, the user may choose an atomic configuration of a sphere, cylinder or rectangular prism and the corresponding length scale parameters. If the user specified geometry spans multiple grains within the FEM, OFEMD initialises each grain with atoms configured in the lattice orientation retrieved from the FEM. A shell of atoms, indicated by the darker atoms in Figure 5 is used to impose the boundary strains extracted from the continuum simulation.
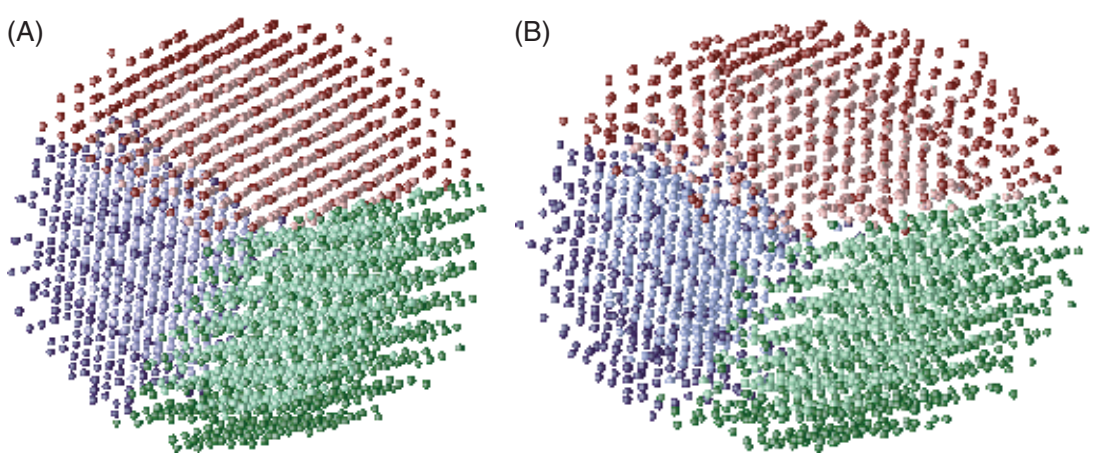

Figure 5: Direct Atomistic Simulation of Triple Junctions. A cylinder of atoms decorates a finite element edge that coincides with a triple junction of grains. (A) shows the initial configuration. (B) shows the atoms after they have been deformed according to the finite element displacements and relaxed, opening a crack along two of the interfaces 
After relaxing the atoms to find a natural configuration, the atoms are deformed according to the mesh displacements solved for by the finite element simulation. The outer shell of atoms are held fixed while the inner atoms are relaxed according to the chosen dynamical algorithm.

Overlapping Finite Elements and Molecular Dynamics can feed back to larger scale, continuum simulations in three ways: with decision-making information such as how a crack propagates at a triple junction of grains, crack initiation parameters and overall comparison with small scale continuum simulations. If a crack nucleation, branching or turning event is found, the continuum simulation can be updated, and the crack grown with continuum models such as the CZM.

To further improve on the fidelity of polycrystal fracture simulations, OFEMD could be used with a tool such as OOF $[25,26]$ which creates finite element simulations directly from experimental microstructure data. The result will be the capability to perform fully atomistic, fully continuum, or multiscale fracture simulations based on realistic geometries that can be compared directly to experiment.

\section{Conclusion}

We have discussed three main challenges involved in continuum modelling of polycrystal fracture. First, exploring the cohesive properties of $3 \mathrm{D}$ grain boundaries involves exploring a 5D space. Second, the peak stress and energy have singularities at all commensurate grain boundaries. Even if it were not for the first two challenges, our comparisons of atomistic and finite element simulations of polycrystal fracture show that cohesive properties of the interfaces alone are not enough to model the fracture of polycrystals using continuum methods. Sites such as triple junctions, edges and corners of grains are important nucleation sites. To resolve this last challenge, we suggest the use of direct atomistic modelling of local regions of interest.

\section{ACKNOWLEDGEMENTS}

This work was supported by NSF Grants No. ITR/ASP ACI0085969 and No. DMR-0218475. We also thank Gerd Heber, Drew Dolgert, Mu Liu, Surachute Limkumnerd, Chris Myers and Paul Wawrzynek.

\section{REFERENCES}

1. Shenoy, V. B., Miller, R., Tadmor, E. B., Rodney, D., Phillips, R. and Ortiz, M. (1999) An adaptive finite ele- ment approach to atomic-scale mechanics: the quasicontinuum method. J. Mech. Phys. Solids 47, 611-642.

2. Needleman, A. (1990) An analysis of tensile decohesion along an interface. J. Mech. Phys. Solids 38, 289324.

3. Xu, X. P. and Needleman, A. (1994) Numerical simulations of fast crack growth in brittle solids. J. Mech. Phys. Solids 42, 1397-1434.

4. Camacho, G. T. and Ortiz, M. (1996) Computational modelling of impact damage in brittle materials. Int. J. Solids Struct 33, 2899-2938.

5. Tvergaard, V. and Hutchinson, J. W. (1992) The relation between crack growth resistance and fracture process parameters in elastic-plastic solids. J. Mech. Phys. Solids 40(6), 1377-1397.

6. Coffman, V. R., Sethna, J. P., Heber, G., Liu, A., Ingraffea, A. R. and Barker, E. I. (2008) A comparison of finite element and atomistic modeling of fracture. Modelling Simul. Mater. Sci. Eng. 16, 065008 (15 pp).

7. Iesulauro, E., Ingraffea, A. R., Arwade, S. and Wawrzynek, P. A. (2002) Simulation of grain boundary decohesion and crack initiation in aluminum microstructure models. In Fatigue and Fracture Mechanics, Vol 33 (W. G. Reuter and R. S. Piascik, Eds). American Society for Testing and Materials, West Conshohocken, PA, 715-728.

8. Iesulauro, E., Ingraffea, A. R., Heber, G. and Wawrzynek, P. A. (2003) A multiscale modeling approach to crack initiation in aluminum polycrystals. In 44th AIAA/ASME/ ASCE/AHS Structures, Structural Dynamics, and Materials Conference. AIAA, Norfolk, VA.

9. Warner, D. H., Sansoz, F. and Molinari, J. F. (2006) Atomistic based continuum investigation of plastic deformation in nanocrystalline copper. Int. J. Plast. 22, 754-774.

10. Yamakov, V., Saether, E., Phillips, D. R. and Glaessgen, E. H. (2006) Molecular-dynamics simulation-based cohesive zone representation of intergranular fracture processes in aluminum. J. Mech. Phys. Solids 54, 1899-1928.

11. Yamakov, V., Saether, E. and Glaessgen, E. H. (2008) Multiscale modeling of intergranular fracture in aluminum: constitutive relation for interface debonding. J. Mater. Sci. 43, 7488-7494.

12. Zhou, X. W., Moody, N. R., Jones, R. E., Zimmerman, J. A. and Reedy, E. D. (2009) Molecular-dynamics-based cohesive zone law for brittle interfacial fracture under mixed loading conditions: effects of elastic constant mismatch. Acta Mater. 57, 4671-4686.

13. Coffman, V. R. and Sethna, J. P. (2008) Grain boundary energies and cohesive strength as a function of geometry. Phys. Rev. B 77, 144111.

14. Bozek, J. E., Hochhalter, J. D., Veilleux, M. G. et al. (2008) A geometric approach to modeling microstructurally small fatigue crack formation-part I: probabilistic simulation of constituent particle cracking in AA 7075-T651. Modelling Simul. Mater. Sci. Eng. 16, 065007 (28 pp).

15. Hochhalter, J., Littlewood, D., Veilleux, M., Bozek, J., Ingraffea, A. R. and Maniaty, A. (2009) A geometric approach to modeling microstructurally small fatigue crack formation: II. simulation and prediction of crack 
nucleation in AA 7075-T651. Modelling Simul. Mater. Sci. Eng. accepted for publication. http://www.iop.org/EJ/ journal/ -age=forthart/0965-0393.

16. Wolf, D. and Yip, S. (Eds) (1992) Materials Interfaces: Atomic-level Structure and Properties. Chapman \& Hall, London.

17. Sansoz, F. and Molinari, J. F. (2005) Mechanical behavior of $\sigma$ tilt grain boundaries in nanoscale $\mathrm{cu}$ and al: a quasicontinuum study. Acta Materialia, 53:1931-1944.

18. Chen, S. P., Srolovitz, D. J. and Voter, A. F. (1989) Computer simulation on surfaces and [001] symmetric tilt grain boundaries in ni, al, and ni $3 a \mathrm{l}$. Journal of Materials Research, 4:62.

19. Chadwick, G. A. and Smith, D. A. (Eds) (1976) Grain Boundary Structure and Properties. Academic Press Inc., London.

20. Bailey, N., Cretegny, T., Sethna, J. P., Coffman, V. R., Dolgert, A. J., Myers, C. R., Schiotz, J. and Mortensen, J. J. (2006) Digital material: a flexible atomistic simulation code. Available at http://arxiv.org/abs/cond-mat/0601236.
21. Baskes, M. I., Nelson, J. S. and Wright, A. F. (1989) Semiempirical modified embedded-atom potentials for silicon and germanium. Phys. Rev. B 40, 6085-6099.

22. Baskes, M. I. (1992) Modified embedded-atom potentials for cubic materials and impurities. Phys. Rev. B 46, 2727.

23. Heber, G. and Gray, J. (2005) Supporting finite element analysis with a relational database backend; part i: there is life beyond files. Technical report, Microsoft Research.

24. Heber, G. and Gray, J. (2006) Supporting finite element analysis with a relational database backend part ii: database design and access. Technical report, Microsoft Research.

25. Reid, A. C. E., Langer, S. A., Lua, R. C., Coffman, V. R., Haan, S. and García, R. E. (2008) Image-based finite element mesh construction for material microstructures. Comput. Mater. Sci. 43, 989-999.

26. Reid, A. C. E., Lua, R. C., García, R. E., Coffman, V. R. and Langer, S. A. (2009) Modelling microstructures with oof2. Int. J. Mater. Prod. Technol. 35, 361-373. 